\title{
Information Structure of STEP Convertor of STEP-CNC Mapping System
}

\author{
Noordiana Kassim ${ }^{1, a}$, Yusri Yusof ${ }^{2, b}$, Ida Aryanie Bahrudin ${ }^{3, c}$, \\ Rafizah Mohd Hanifa, ${ }^{4, d}$, Mohd Hadri Mohamed Nor ${ }^{5, e}$, \\ Azizul Rahman Abd Aziz ${ }^{6, f}$ and Zahrul Hisham Othman ${ }^{7, g}$ \\ ${ }^{1}$ Center for Diploma Studies, University Tun Hussein Onn Malaysia, Batu Pahat, Johor \\ ${ }^{2,7}$ Faculty of Mechanical and Manufacturing Engineering, University Tun Hussein Onn Malaysia, \\ Johor, Malaysia \\ ${ }^{3,4,5,6}$ Center for Diploma Studies, University Tun Hussein Onn Malaysia, Batu Pahat, Johor
}

anoordiana@uthm.edu.my, ${ }^{b}$ yusri@uthm.edu.my, caryanie@uthm.edu.my, ${ }^{\text {d }}$ rafizah@uthm.edu.my,

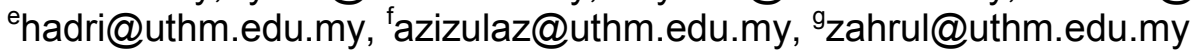

Keywords: ISO 10303, ISO 14649, STEP, STEP-NC.

\begin{abstract}
ISO 14649 standard, commonly recognized as STEP-NC is a data model interface for computer numerical controllers. STEP-NC goes much further that $\mathrm{G}$ codes and has a more comprehensive data model that overcomes the lack of process information in ISO 6983 files (G codes programming). The STEP compliant approach brings the possibility to integrate the machine controller to the overall enterprise information network, with the intention of having bi-directional data flow. This will provide the novel capability for upstream feedback of information from the numerical controller to the design and process areas in the process planning and manufacturing. This paper presents the key concepts of the proposed STEP Convertor of the STEP-CNC Mapping System. The objective of the main system is to take a generic STEP file as the input to drive a CNC machine tool. The main system is broken down into two main sub-systems, STEP Convertor and STEP-NC Translator. This paper will focus solely on the information structure and conceptualization of the STEP Convertor.
\end{abstract}

\section{Introduction}

Various studies have been conducted to enhance the capabilities and expanding the applications of machining simulation. Till now, a variety of approaches and methods have been developed and utilized to simulate manufacturing processes [1]. Most of STEP-NC research objectives are to prove the completeness and robustness of the STEP-NC data models, and to investigate ways of deploying STEP-NC data on a CNC machine tool [2].

The purpose of a product model is to provide a means for representing and exchanging information about a product gathered during, and used in, the design and manufacture of that product. Therefore, the contents of this product model must be able to support the information needs of a large variety of computerized manufacturing applications.

The popularity of using CAD systems as a means for creating, representing and exchanging product designs has created various standard product data exchange formats and have shown a success in transferring data between CAD systems, but they have failed to transfer product data from CAD to CAM applications. This is because current CAD systems are not able to support all the information concerning a part that is needed to support the CAM activities [3]. One solution that offers the most promise is the creation of a standard method for representing product data that would serve the needs of all applications. The Standard for the Exchange of Product model data (STEP) is such a standard, offering the most promise as an answer to these problems [4]. STEP-NC is intended to be a replacement for $\mathrm{G}$ codes that provides much richer data and allows feedback of process information from the machine tool back upstream to design and process planning [5]. 
STEP-NC is currently being validated in research projects, and is showing promise as the next generation data interface to machine tools.

The main focus of this research paper is on the concept and architecture development for the proposed STEP Convertor of the STEP-CNC Mapping System. Based on studies on previous research it was revealed that the majority of the existing STEP-NC enabled systems are about deploying a STEP-NC data model for a particular type of CNC controller or machine tool. STEPNC's true benefit of enabling bidirectional machining information data flow have not been sufficiently exhibited nor proved. For a CNC machine to run on STEP-NC data, the controller will have to first process the STEP-NC data and adapt it to the machine tool.

\section{STEP-NC}

The nature of the STEP-NC data model lies in the fact that its main purpose is to document manufacturing tasks of what to do information, instead of method how to do. Thus its comprehensive bidirectional data exchange is guaranteed, through its object oriented design and the use of data elements of the widely disseminated STEP standard (ISO 10303). For a CNC machine to run on STEP-NC data, the controller will have to first process the STEP-NC data, adapt it to the machine tool and even better make intelligent, on-line and real-time machining decisions at execution.

Different technical process planning aids have different levels of automation and scope with respect to process planning activities. For a better overview, a summary is given according to use in the product realization chain in Fig 1. Therefore, there are two tasks that need to be accomplished, faithful interpretation of STEP-NC data and execution of the STEP-NC data on a machine tool. These are also the main tasks of this research.

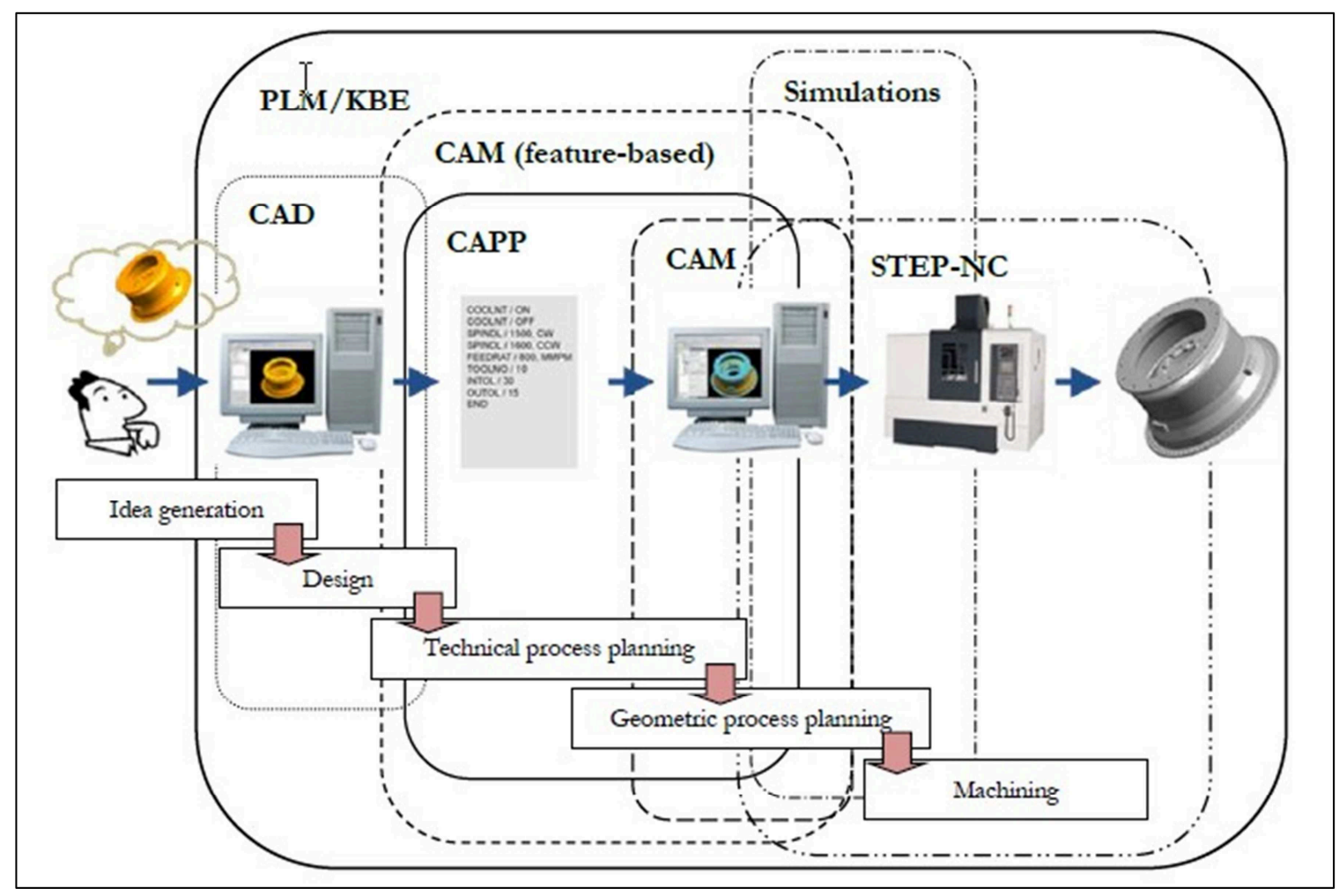

Fig 1 Overview of Process Planning and Their Area of Applicability (Anderberg, 2012)

\section{Methodology of the Research}

The high-level data in the STEP-NC data model contains information such as machining features, set-up, Workingstep and etc., all of which is defined based on a set of schema in 
EXPRESS. These data however cannot be directly used on a machine tool without modifications. The low level data that is purposely prepared for a particular machine tool must be generated from the high-level STEP-NC data. This low-level data can then be further processed (into G-code for example) for final execution on a machine tool. The dominance of G-code in the CNC systems means that most of the CNC controllers still run on G-codes.

This means that there are two tasks in hand in this research, and they correspond to two processes to be carried out. Firstly, the generated STEP-NC data needs to be "adapted" to the specific machining environment (e.g. the type of machine tool, cutting tools, etc.) so that a native version of STEP-NC data can be obtained. Secondly, generation of G-codes that are ready to be executed on a machine tool is needed.

The module responsible for the first process is hereby called STEP Convertor, whereas that for the second process is called STEP-NC Translator. STEP Convertor and STEP-NC Converter can be embedded in the controller software. This way, the STEP-NC data can be made portable across different CNC systems as long as they have the STEP Convertor and STEP-NC Translator. This research paper will focus solely on the STEP Convertor within the milling process of the CNC machining.

The development of a generator called STEP Convertor that can populate or repopulate a generic STEP file to be a native STEP-NC file is the main task of this research paper.

\section{The STEP Convertor}

When a STEP-NC file is generated at the process plan phase like design, it contains only information about common manufacturing data such as machining strategies on how to machine the part, the decisions as to where to manufacture the part and what manufacturing facilities to use are still pending at that stage.

STEP Convertor is to adapt this generic STEP file to a native STEP-NC by populating, instantiating or customizing the generic STEP file, taking into consideration of the targeted manufacturing facilities, like machine tool and cutting tools. This convertor can also convert a native STEP-NC file to a different manufacturing environment.

During initial stage of developing the STEP Convertor, it is necessary to understand the specific shop-floor plan information data in order to figure out what information in a STEP-NC file is to be populated if the STEP-NC file is a generic one, or re-populated if the STEP-NC file is a native one already. The type of shop-floor information needed are usually related to the workpiece, machine tools, set-up information, cutting tools available, and machining functions and machining strategies. Fig 2 shows the logical structure of the STEP Convertor.

Workpiece Information. Machining is any of various processes in which a piece of raw material is cut into a desired final shape and size by a controlled material-removal process. There are many different types of raw materials that are considered for this research.

When the raw material for a workpiece has been decided and selected, it needs to be documented in the STEP-NC file. The size of workpiece will affect a number of entities defined in STEP-NC, for example the Manufacturing_feature, the fixtures and clamping positions. The property of material on the other hand, affects entities such as Manufacturing_function such as feed-rate and spindle speed, and Manufacturing_strategy such as cutting_depth, step_over and overlap. 


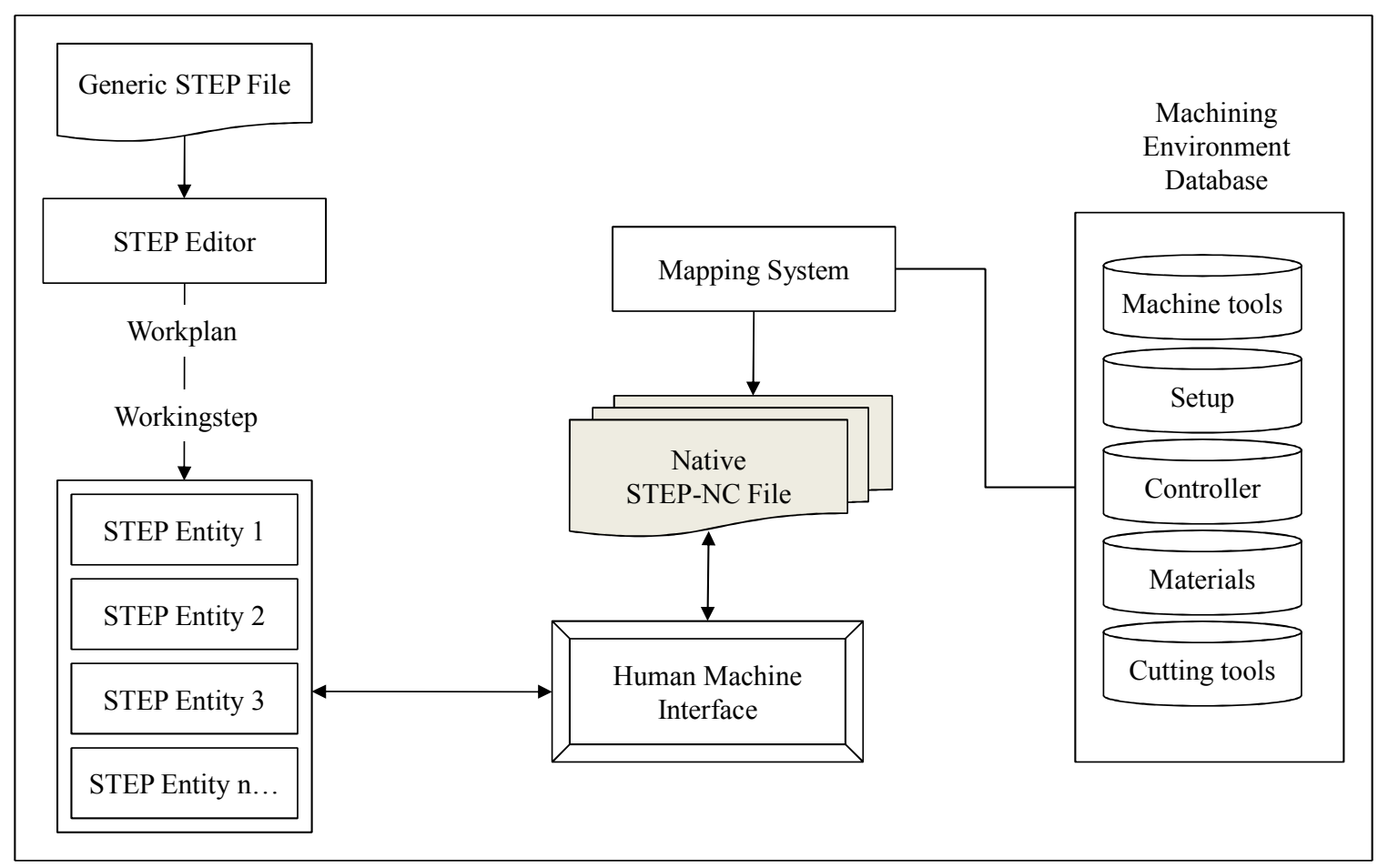

Fig 2 Logical Structure of STEP Convertor.

Machine Tools Information. Machine tools employ some sort of tool that does the cutting or shaping. All machine tools have some means of constraining the workpiece and provide a guided movement of the parts of the machine. Thus the relative movement between the workpiece and the cutting tool (which is called the toolpath) is controlled or constrained by the machine. Several information regarding machine tools need to be gathered before the actual machining process could take place like the size and the structure of the machine tool. This information will be used to populate the generic STEP file data. If more than one machine tools is needed for the job, the best decision will need to be make on deciding the best possible choice available. It is very likely that for a complex machining process, it would require more than just one machine tool, and multiple machine tools are outside the scope of this research.

Setup Information. The set-up information is mainly to do with its location on the machine and how the workpiece is clamped. More specifically, the set-up information will populate STEP-NC entities such as set-up coordinate system, workpiece coordinate system and feature coordinate system.

Cutting Tools Information. A cutting tool or cutter is any tool that is used to remove material from the workpiece. Cutting may be accomplished by single-point or multipoint tools. Cutting tools must be made of a material harder than the material which is to be cut, and the tool must be able to withstand the heat generated in the metal-cutting process.

The cutter data in a STEP-NC file needs to reflect what is available in a tool magazine or tool turret. What was defined in a STEP-NC file may not be same as the preloaded cutters on the machine. The cutter information affects STEP-NC entities such as machining technology (feedrate, cutting speed), machining_strategy (cutting_depth, step_over and finishing allowance), and even Workplan (the sequence of operation).

Machining Function and Strategies Information. In STEP-NC, this information is kept in entity Machine_function. Machine_function is machine tool dependent. It indicates the state of various machine functions, like coolant, chip removal, etc. to be applied during the time span of this operation. The original STEP-NC file may define an operation with the coolant-through function, which may not be available 


\section{Summary}

As intended from the beginning, STEP-NC is to remain as a high-level data model that contains primarily generic design and manufacturing information independent of machine tools. The STEP Convertor is responsible for generating a native program that contains the information about the targeted machine tool in a STEP-NC format from a generic STEP file generated during the design process; make it from AutoCAD or Solidworks software as an example. Therefore, it populates a generic STEP program with the native data obtained from design stage and internal databases to obtain a native version of the STEP-NC program.

\section{References}

[1] A. Abdul Kadir and X. Xu, "Towards High-Fidelity Machining Simulation " Journal of Manufacturing Systems, p. 12, 2011.

[2] H. Wang, "New Control Strategy for CNC Machines Via STEP-NC," Doctor of Philosophy, Department of Mechanical Engineering, University of Auckland, Auckland, New Zealand, 2009.

[3] Y. Yang, et al., "The Role and Application of STEP in CAD/CAPP/CAM integration," presented at the IEEE TENCON, Beijing, 1993.

[4] S. M. Amaitik and S. E. Kilic, "STEP-based Feature Modeler for CAPP," presented at the Proceeding of ICRM-2002 Conference Gaziantep, Turkey, 2002.

[5] S. T. Newman, Allen, R. D. and Russo Jr., R. S. U. , "CAD/CAM Solutions for STEP Compliant CNC Manufacture," in Proceedings of the 1st CIRP(UK) Seminar on Digital Enterprise Technology, School of Engineering, University of Durham, 2002. 